\title{
La reconstrucción de la memoria: la poesía perua- na después de la violencia política 2000-2010
}

\section{Reconstructing Memory: Peruvian Poetry After Political Violence 2000-2010}

\author{
Carlos Villacorta \\ University of Maine \\ carlos.villacorta@maine.edu

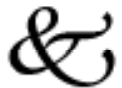

Resumen

El presente trabajo analiza la última poesía peruana que articula y representa la violencia política que sufrió la sociedad peruana durante los años ochentas y los años noventas. Durante esas dos décadas, el lenguaje conversacional, considerado hegemónico por mucho tiempo en el escenario poético peruano, fue perdiendo legitimidad ya que no fue suficiente herramienta para retratar la vertiginosa atomización de la sociedad. Al mismo tiempo, la poesía peruana empezó un largo proceso de reformulación y de búsqueda de un nuevo lenguaje durante la década de los noventas que se fue acentuando al inicio del siglo XXI. Los trabajos de una nueva generación de poetas como Victoria Guerrero, Luis Fernando Chueca, Roxana Crisólogo, entre otros, han tenido una doble preocupación: reformular el lenguaje poético peruano y representar lo que fue el Conflicto Armado interno (1980-2000) ya sea desde sus causas, proceso y/o consecuencias.

Palabras claves: Poesía peruana, Conflicto Armado Interno, poesía conversacional, 


\begin{abstract}
This paper analyzes the last Peruvian poetry that articulates and represents the political violence suffered by Peruvian society during the eighties and nineties. During those two decades, conversational language, long considered hegemonic in the Peruvian poetry scene lost legitimacy because it was not enough to portray the fast atomization of society. At the same time, Peruvian poetry began a long process of reformulation and search for a new language during the nineties that was accentuated at the beginning of the century. The works of a new generation of poets like Victoria Guerrero, Luis Fernando Chueca, Roxana Crisólogo, among others, have had two concerns: reformulate Peruvian poetic language and represent what was the Internal Conflict (1980-2000) whether from its causes, process and / or consequences.
\end{abstract}

Keywords: Peruvian poetry, Internal Conflict Army, Conversational poetry.

Recibido: 15/5/16 Aceptado: 20/6/16

En el año 2000, la Comisión de Derechos Humanos del Perú, junto con el grupo de teatro Yuyachkani, publicó la obra que propusieran al poeta José Watanabe. El libro en cuestión era una personal adaptación de la tragedia Antígona, del dramaturgo griego Sófocles. La historia, por todos conocida, narra el conflicto entre la joven Antígona y el rey Creonte al término de la guerra en Tebas: sus dos hermanos Polinices y Eteócles han muerto y sólo uno (Eteócles) puede ser enterrado en la ciudad, pues éste ha defendido Tebas, mientras que el primero, por alzarse contra Creonte, es un traidor que no merece ser enterrado en ninguna parte. Siguiendo la historia original, Watanabe elabora un texto que no es esencialmente una obra teatral clásica, sino que, usando distintos personajes, construye poemas que juntos van desarrollando la tragedia.

Antígona, en la versión de Watanabe, fue escrita para que una única actriz interpretara todos estos papeles: la narradora, Antígona, Creonte, Tiresias, Hemón, un soldado e Ismene. ${ }^{1} \mathrm{Al}$ mismo tiempo, Watanabe construyó el texto con el fin de revelarnos que quien narra la tragedia no es otra que la hermana de Antígona, Ismene, quien se rehúsa a ayudar a su hermana a enterrar al hermano abandonado. La obra/poemario termina con la verguienza de Ismene, quien reconoce su error y pide a su hermana que interceda por ella para ganar el perdón de Polinices.

1 La actriz fue la peruana Teresa Rally. 
Antígona es un texto importante porque fue llevado a escena en 2001 por la sierra peruana, así como por la capital. No se trata del primer texto que retrata la violencia política en Perú entre 1980-2000. Pero es el primer texto que propone el problema del sujeto de la post-guerra. Antígona presenta dos preguntas a toda una nación: por un lado, como testigos, qué significó la guerra o la violencia que ha sufrido el país; por otro, como sobrevivientes, qué hacer con los fallecidos, con aquellos que murieron durante el conflicto de diversas maneras.

En el mismo año, se creó la Comisión de la Verdad y Reconciliación, con la finalidad de crear un informe que diera cuenta de veinte años de violencia en el territorio peruano; es decir, de las causas, el proceso, así como las consecuencias de dicha violencia. Los resultados presentados en 2003 fueron estremecedores: cerca de 70 mil muertos, responsabilidad política de tres diferentes gobiernos (el de Fernando Belaúnde Terry, Alan García y Alberto Fujimori), así como responsabilidad directa de las Fuerzas Armadas del Perú, el movimiento maoísta Sendero Luminoso y el Movimiento Revolucionario Túpac Amaru (MRTA).

Con este fondo político y social, ¿qué sucedió a partir de ese momento con la poesía peruana? ¿Cómo se acercó al tema de la guerra? ¿Cuáles fueron sus nuevas líneas? ¿Las hubo? Para responder esto, sería necesario un ensayo específico sobre cada momento anterior para ver si existen líneas poéticas que continúan o no. Sin ánimo de ser simplista, trataré de hacer un esbozo de esa situación previa.

En los ochentas, el optimismo de los setentas ha desaparecido. Con un país que ha dejado atrás la dictadura militar de los setentas para entrar en la desastrosa democracia de los ochentas, los jóvenes peruanos, entonces, ven el presente como un fracaso. En ese momento, aparece en Lima el grupo poético Kloaka. Como bien afirma la crítica Giancarla Di Laura sobre ellos:

... el movimiento Kloaka es una respuesta a la parametrada clase burguesa cuyos falsos presupuestos ideológicos eran vistos como verdades absolutas durante [los ochentas] en el Perú. Asimismo, la postura de Kloaka fue de enfrentamiento ante el poder establecido cuyos actos fueron cuestionados por la irrupción de una diversa gama de violencia política, principalmente el MRTA y el PCP-Sendero Luminoso.

Las búsquedas poéticas de este grupo deben relacionarse con las preocupaciones estéticas de la generación de los sesentas y setentas, quienes empezaron a hacer una revisión histórica de aquello denominado como lo peruano. En el caso de la década de los sesentas, poetas como Antonio Cisneros, Luis Hernández, Javier Heraud y Rodolfo Hinostroza presentaron en sus diversas 
propuestas un país que aún se estaba construyendo, así como la preocupación por llevar a cabo una revolución de tipo socialista en el Perú. A estas propuestas, los poetas de los setentas (especialmente los del grupo poético Hora Zero) buscaron incorporar a las nuevas masas a la discusión. Pero ya a mitad de los setentas y claramente en los ochentas, todo ese proceso empieza a desmoronarse y los nuevos jóvenes se dan cuenta de que el Perú vive un proceso acelerado de destrucción de aquello que no llegó a crearse: la nación peruana. ${ }^{2}$

No hay que olvidar que el cuestionamiento de la peruanidad implicó también el cuestionamiento de las formas poéticas, especialmente el del lenguaje de las vanguardias de primera mitad del siglo XX. La búsqueda de un nuevo lenguaje significó inicialmente utilizar un discurso coloquial o conversacional. Sobre este punto, Antonio Cornejo Polar sostiene: “... lo que sucede es que el poeta abandona la posición de hablante, o la comparte y colectiviza, para ocupar otra: la de constructor de un texto múltiple, polifónico, que remite a varios sujetos cuyas identidades, ficticias o no, desplazan al poeta y lo sitúan más allá del tejido de los discursos que constituyen el texto" (1980: 205).

La pregunta es hasta qué punto la forma conversacional permitía dar cuenta de las necesidades expresivas de cada poeta así como las del tejido social al que buscaban representar sin entrar en una suerte de callejón sin salida. Para Cornejo: "Mucho más que constituirse como síntesis iluminadora de la nacionalidad o de toda nuestra América, la poesía conversacional intenta extenderse por al ambiguo y difuso sentir comunitario, recogiendo lo que es común y negándose a elevarlo a la condición de símbolo o emblema de categorías mayores" (1980: 204).

Como mencioné, la destrucción de ese sentir comunitario se hizo patente en la década de los ochentas, en los que la sociedad se vio afectada por diversos discursos que literalmente fueron dinamitando los lazos colectivos que tan sólo unos años atrás habían aparecido con la promesa de formar un nuevo país. Los poetas de los ochentas dan cuenta de ello desde distintas perspectivas: periféricas (Domingo de Ramos, Róger Santiváñez), feministas (Rocío Silva Santisteban, Mariela Dreyfus), entre otras. El lenguaje conversacional empezó poco a poco a verse como una forma insuficiente para explicar lo que estaba más allá del lenguaje: el miedo, la violencia, la destrucción. Esta sensación es

2 En realidad, los mismos textos de los poetas de los sesentas van presentando cierto pesimismo acerca de una posible revolución. Socialmente, algunos hechos van confirmando esta sensación: la muerte del poeta Javier Heraud en la selva peruana (1963), la desaparición del Movimiento de Izquierda Revolucionaria (MIR) en 1965. Contra lo esperado, la famosa revolución llegaría de la mano de los militares, quienes al mando del general Juan Velasco Alvarado inauguraron el Gobierno Revolucionario de las Fuerzas Armadas en 1968. 
clara al inicio de los noventas, donde el tiempo y el espacio se han detenido, dando así un claro sentimiento de encierro y de vivir en un limbo. La joven poeta Montserrat Álvarez lo expresa en uno de sus versos de su primer poemario publicado en 1990, Zona Dark:

En estos días en los que la muerte es un adorno para la vida, las horas del futuro se han venido al presente; los relojes se han roto, o se los han robado. (Villacorta 2005: 21)

Los noventas comienzan con este espacio detenido, con este tiempo que no pertenece a los nuevos jóvenes. Estos irán participando de una sociedad que sufre la aplicación del modelo neoliberal y autoritario de Alberto Fujimori durante los diez años que durará su gobierno. El crítico y poeta peruano Luis Fernando Chueca se ha referido a esta década como el momento de una "Consagración de lo diverso". En este ensayo de 2001, Chueca propone la diversidad de propuestas y de formas poéticas como un sello distintivo de esta última década. Al mismo tiempo, hace hincapié que muchas (si no todas) son formas que aparecieron en la diversidad de la década del setenta, es decir, durante la aparición del grupo Hora Zero. Sin embargo, esa diversidad que parte de la sensación de inercia con la que empieza la década oculta en el fondo un alejamiento a retratar directamente el tema de la violencia política y social que se vivió durante ese mismo período. Entonces, se pregunta el crítico:

[...] cómo la guerra interna empapó los ánimos de una promoción de poetas que participó, al menos -más o menos cerca, más o menos lejosdel embrollo subjetivo producido y cómo se trasluce eso en la poesía. La desazón y el escepticismo reflejados en los textos, el regreso a la revisión de los conflictos de la intimidad y los quiebres internos (exilios interiores, podríamos decir, como único refugio frente a un mundo que se desmorona) o la recuperación poética de los espacios familiares, que se erigen como panteones privados que se contraponen a la percepción de lo colectivo nacional nos dan algunas pistas, aunque, claro, no es posible querer explicar estos caminos sólo como reacciones a lo experimentado frente al espacio público. También se puede señalar que los poetas de los 90 , al verse desbordados por acontecimientos como los de la violencia política, que exigían una distancia crítica mayor, pues superaban las posibilidades aún en formación de sus lenguajes, prefirieron evitarlos. $\mathrm{O}$ decir que la costumbre de la muerte que produce corazas infranqueables, incluso en la más permeable subjetividad de los poetas. Otras interpretaciones apuntan a que los acontecimientos de la guerra que desangró al país pasaron lo suficientemente lejos de los poetas, que, en tal sentido, no se sintieron empujados a procesar desde sus textos lo vivido en otros 
ámbitos, o a la postulación de un movimiento de retracción frente a la intensidad de un discurso que estuvo muy atento al espacio público y a las diversas violencias como escenario poético fundamental (y quizás aquí se encuentren, parcialmente, algunas de las explicaciones de la pérdida de la hegemonía de la poesía conversacional). (Chueca 2001: 117).

Efectivamente, el panorama poético del siglo XX en Perú se cierra con una sensación de atomización de la sociedad y de la pérdida de un lazo de comunidad o nación. Lo que Chueca finalmente muestra en su estudio es el estado de individualización en el que se ha quedado la sociedad peruana luego de dos décadas de cambio social. Asimismo, aunque trata de encontrar propuestas que unan a los dispersos jóvenes poetas de los noventas (Chueca reconoce al menos nueve espacios o lugares por donde transita esta generación), la sensación de vacío persistió y moldeó el sentir de mucho de ellos.

Como mencioné al principio, la aparición de un texto como Antígona, la caída de la dictadura fujimorista, el pase a la democracia y finalmente la creación de la Comisión de la Verdad y Reconciliación son hechos concretos de que se viven otros tiempos en Perú. A partir de la entrega del informe de la comisión, los escritores comienzan a escribir sobre la violencia social y política no sólo de los últimos veinte años, sino incluso desde mucho antes, desde los sesentas.

A partir de 2004, poetas de distintas generaciones afrontan el tema casi directamente. Esa revisión histórica aparece, por ejemplo, en dos poetas de distinta edad, pero de la misma generación, los noventas. Victoria Guerrero publica en el 2005 su cuarto poemario, Ya nadie incendia el mundo. En él, la historia nacional se engarza con la historia personal de la poeta. La escritura del yo poético se enmarca en el mismo momento del nacimiento (el año 1971) en un Hospital del Estado. De esta manera, historia nacional y yo poético no son dos sujetos disociados uno al margen del otro, sino que el nacimiento de uno inscribe el nacimiento de otro. De esta manera, la poeta noventera es literalmente hija de la década de los setenta (del cambio social aplicado por el general Velasco) y de la poesía del mismo tiempo (especialmente de la poeta Carmen Ollé). Es interesante notar que esta inscripción es la de un lenguaje que "no sana el cuerpo". Este cuerpo es el de una niña que nace a los siete meses y tiene que vivir dentro de una "máquina-madre" (metáfora para incubadora) mientras escucha a los otros (¿los médicos?, ¿la sociedad?) decir: "niña idiota / -dijeron / aspira más allá de tu cuerpo defectuoso y lárgate ya no tenemos espacio para ti ni para los tuyos" (Guerrero 2005: 20). El nacimiento se acepta y la incorporación del recién nacido se incluye en el discurso nacional, pero siempre desde la negación: "no tenemos espacio para ti ni para los tuyos". La comparación resulta evidente. La movilización social aplicada por el gobier- 
no de Velasco intentó integrar a todos los ciudadanos que no habían tenido participación real en Perú y que, en muchos casos, no eran considerados ni ciudadanos. Eventualmente, el mismo aparato del Estado no pudo hacer frente a las distintas necesidades de la nueva sociedad peruana a la que buscaba representar. Dentro de este contexto, el nacimiento se produce en un tiempo que inscribe al nuevo ciudadano para abandonarlo a su suerte. Por este motivo, el abandono del recién nacido puede leerse como la ausencia de un padre (el Estado) incapaz de hacerse cargo de sus nuevos ciudadanos.

El año siguiente, Manuel Fernández publica su primer libro Octubre. El nombre también alude a un origen de la violencia nacional: el 3 de octubre de 1968 el general Juan Velasco Alvarado tomó el poder. Esta historia, que es la historia de los padres del poeta, se encadena con la historia del poeta en los noventas donde “LA SÓLIDA BELLEZA DE LOS TANQUES” (2006: 20) parecen lo único real y verdadero bajo el siempre gris cielo de la capital. La violencia, los apagones, los cortes de luz, son algunas de las situaciones que parecen no haber cambiado. Es más, para Fernández, en su particular lectura de la historia peruana, del golpe de Velasco al de Fujimori en $1992^{3}$ hay un único tiempo donde lo demás, incluso el lenguaje, es una ilusión: "ERES ESCULTURA HECHA CON ARENAS DE PLAYAS INEXISTENTES" (2006: 28). Y todo ese tiempo de 22 años parece el tiempo de embarazo que ha dado a luz a un nuevo ser, aunque no sepamos a quién. "Esperamos respuestas todavía", sentencia el poeta al final de su libro. En Guerrero y Fernández, entonces, el lenguaje poético aparece como algo defectuoso e incluso ilusorio. Sin embargo, ese es el poder que permite inscribir la memoria misma del país.

En otro aspecto, las poetas Roxana Crisólogo y Rocío Silva Santisteban buscan retratar al sujeto femenino de esta época. En el caso de la primera, su tercer libro, Ludy $D$, recrea la vida de una compañera de estudios de la poeta. Ludy D es una joven que llega a Lima desde Huánuco en los setentas. Durante los ochentas, dejará sus estudios universitarios para unirse a Sendero Luminoso. El poemario, dividido en tres partes con poemas sin títulos, se presenta como un diario poético que busca dar cuenta de la vida de una joven migrante que vive la pesadilla que es el país. El primer poema es sintomático de esa desesperanza y de esa batalla interminable que es la supervivencia en Perú: "no había agua para regar un jardín / el desierto era aquella humanidad / y el polvo / que mi madre empuja con la escoba." (2006: 8) La imagen del desierto revela esa comunión con el todo (¿la nación migrante?) que al llegar a casa se desintegra

3 El 5 de abril, Alberto Fujimori produjo un autogolpe con apoyo de las Fuerzas Armadas. Los tanques volvieron a la calle frente al Congreso de la República para evitar que cualquier diputado o senador pudiera entrar. 
en polvo, en carencia, y se transforma en un trabajo interminable semejante a la infinita escasez de agua.

Rocío Silva Santisteban, con su libro Las hijas del terror (2007), se enfrenta a aquellos que violentan al otro, específicamente al sujeto femenino. El poema inicial, "iLe tienes miedo a la sangre?", clarifica su posición frente al lector: "Yo no, / vivo con la sangre / la toco, la veo. La huelo / cada mes. No se equivoca" (2007: 11). Efectivamente, el sujeto femenino es consciente de su poder frente a aquel que teme a la sangre. Este sujeto se define entonces por su cercanía a la violencia que hace que emane de su cuerpo la sangre, líquido constitutivo de su identidad y de su poder para enfrentar a la autoridad. Para Silva Santisteban, la escritura de la violencia es básicamente una de género: el hombre, el soldado o militar, es el que inflige dolor y trauma en el otro sujeto, principalmente a la mujer. Según el Informe Final de la Comisión de la Verdad, el $20 \%$ de las víctimas fueron mujeres de todas las edades. Del total de todas estas víctimas, el $97.98 \%$ fue violada, $19.29 \%$ fue torturada, un $25.64 \%$ sufrió algún tipo de herida o lesión y el $14.93 \%$ fue desaparecida. ${ }^{4}$ Esta situación ha terminado creando sujetos totalmente aterrorizados cuyas heridas, lesiones y traumas aún no han sido curados y cuyas secuelas aún existen en el país actual. Efectivamente, después de veinte años de violencia masculina, han nacido "las hijas del terror".

Los muertos son los sujetos que el poeta Luis Fernando Chueca retrata en su cuarto libro Contemplación de los cuerpos (2005). Cada poema funciona como una fotografía o como un documento donde la memoria se inscribe con el fin de, valga la redundancia, rememorar a todos los muertos vistos por quien escribe. El origen de este trauma es la primera muerte y que en este caso en particular es la del abuelo. Con esta muerte desaparece "esa ley que ordena que los hijos entierr[e]n a sus padres, y a los padres de sus padres" (2005: 11). A partir de este momento, el desfile de muertos vendrá como viene la muerte: desordenada, caótica y en contra de esa ley aparentemente natural. Para Chueca, la muerte no construye un cuerpo social como sucede en el poemario Cementerio general (1989), del poeta horazeriano Tulio Mora. La diferencia radica en que mientras Mora coloca nombre y apellido a cada uno de los sujetos muertos y marginados de la historia nacional, Chueca se aproxima a los muertos para afirmar su extrañeza frente a ella y sus restos (unas llaves, una foto, el mismo cuerpo vacío). Para Chueca, la muerte es un hecho tan insondable como inexplicable, donde al final hasta las palabras pierden su sentido: "Es la muerte acaso una palabra” (49). Efectivamente, al final solo queda contemplar y cantar

4 Comisión de Entrega de la CVR. Hatun Willakuy (2004). Lima: Pontificia Universidad Católica del Perú. 
porque el canto "redime del horror" (2005: 53). Las palabras y el sentido solo vuelven a la vida a través de su propia música: el canto es un río por donde puede fluir todo lo visto y experimentado.

Esta misma idea de la muerte se presente en el poemario Tratado de arqueología peruana, de Roberto Zariquiey. El poemario, dividido en tres partes (una por cada una de las regiones naturales del Perú, a saber costa, sierra y selva), busca reconstruir un país a partir del discurso de la arqueología. Su lenguaje también viene directo de las búsquedas de poetas de los sesentas y setentas, cuando las ciencias sociales tomaron un rol protagónico y se utilizaron incluso en el discurso literario. La costa se define por los restos de culturas antiguas que miradas desde el presente asemejan a esas casas del distrito de Magdalena del Mar en Lima, que también se están cayendo a pedazos. Si bien el recurso de la ironía permite desmantelar el discurso arqueológico académico, me parece que el resultado es desigual si lo comparamos con la segunda parte sobre la sierra. Aquí el pasado de las culturas costeñas desaparece para hacer frente a los recién muertos. Estos muertos desenterrados ya no son los de los restos funerarios del pasado preincaico, sino los muertos hallados en fosas comunes en distritos como Huamanga, Huanta, Víctor Fajardo, etc., entre 1980 y el 2000. El lenguaje, por lo tanto, no puede ser el mismo para referirse a ellos: "los muertos no son colocados en los nichos (exactitud semántica: son arrojados)" (2006: 56). La sierra aparece entonces como ese "rincón de los muertos" (aparentemente nombre en castellano del departamento de Ayacucho).

En Tratado, sin embargo, existe una necesidad del escritor de encontrar algo que esté vivo. Si la costa y la sierra son lugares de destrucción y muerte violenta, la respuesta, aquello vivo que es la búsqueda del escritor, debe estar en el último lugar inexplorado del territorio peruano. La tercera sección, la selva, contiene una larga narración acerca del mítico shipibo Shawán Pico. Si bien el poemario busca entender la realidad peruana, su división en tres espacios geográficos es un poco desfasada para entender la complejidad nacional, sobre todo si nos reducimos a una específica etapa en la historia peruana. Además, habría que complicar esta situación con el poema inicial, donde el marco teórico base del poemario asume la escritura de la siguiente manera: "pensar en el Perú como se piensa en un fantasma”. La pregunta es cómo. ¿Con escepticismo? ¿Con incredulidad? ¿Con asombro? ¿Con fe? Cualquiera que sea la respuesta, asumir la realidad como fantasma implicaría buscar pruebas fehacientes de su existencia anterior. Por ese motivo, Tratado de arqueología peruana encuentra paradójicamente más respuestas en el discurso oral que en las ruinas costeñas o los muertos de las fosas comunes de la sierra. 
Los poetas que hemos presentados no son lo únicos que se acercan al tema de la guerra después del Informe.5 $\mathrm{Al}$ menos parte de la poesía de la primera década del siglo XXI busca enfrentarse directamente a las consecuencias de la violencia que azotó al país y que no ha terminado. Es necesario resaltar que para todos estos poetas el Perú existe como país, al menos a priori. Asumir esa realidad les permite escribir un texto y un cuerpo con cierta narratividad. Es decir, en la medida en que se asume la existencia de un territorio y de una nación tenemos los dos elementos principales de la escritura. El primero de ellos es el papel en blanco (el cuerpo del sujeto), lugar donde se inscribe la violencia. El otro elemento sería propiamente la escritura de cada uno de ellos (la personal aproximación al tema de la violencia nacional a través de un lenguaje). Dependiendo de las necesidades y preocupaciones de cada uno tenemos un panorama diverso de aproximación y de logros estéticos. El problema de la violencia política y social de los últimos treinta años ha dinamitado incluso el propio lenguaje coloquial o conversacional de la década de los sesentas y setentas. Este proceso evidente en los ochentas se diluye y se abre a una escritura mucho más amplia en los noventas, donde no prima un tipo de discurso. En la década del 2000, las preocupaciones estéticas de los poetas tienen un compromiso social casi inexistente en la década anterior. Esta necesidad la hacen suya sobre todo poetas de décadas anteriores, no tanto así, o al menos no necesariamente, poetas más jóvenes. ${ }^{6}$ De todo esto, existe una convivencia de diferentes posturas estéticas y preocupaciones sociales, aunque algunos piensen que solo ha existido en todo este tiempo el mismo lenguaje coloquial de hace más de treinta años.7 Las diversas propuestas el día de hoy, al margen de retratar la violencia de las décadas pasadas, buscan su propio camino e incluso nuevas formas de difusión, como Internet.

5 Alejandro Romualdo, poeta de la generación del cincuenta, en su libro Ni pan, ni circo (2005) también retoma el tema de la violencia.

6 Aquellos denominados poetas de la generación del 2000.

7 Me refiero, por supuesto, al ensayo La hegemonía de lo conversacional, del poeta peruano José Carlos Yrigoyen, donde se esgrime que toda la poesía existente desde el setenta pertenece al registro conversacional. Nos parece que su ensayo es una lectura apresurada de las diversas propuestas de los poetas actuales, muchos de ellos con un solo libro en su haber. Luego de diez años ya con dos pies en el siglo XXI, nos parece poco atinado afirmar a la ligera la "hegemonía" de un único tipo de discurso en la poesía peruana, sobre todo si tenemos en cuenta que para hablar de hegemonía habría que hablar de canon y de "evolución", términos que no ayudan o en todo caso, solo revelan el punto de vista "hegemónico" en el que cree el poeta. Recomiendo a los lectores acercarse a los libros de los nuevos poetas para luego buscar, si es necesario, las explicaciones teóricas relevantes. En todo caso, y como punto final, recomiendo sobre esta polémica la respuesta de Luis Fernando Chueca al ensayo de Yrigoyen iLa hegemonía de lo conversacional? Notas para continuar una discusión. (Ver bibliografía). 
Finalmente, las posibilidades estéticas de cada poeta se asientan sobre una fuerte conciencia acerca de la precariedad del lenguaje para retratar este proceso histórico. En varios casos, el espacio de la muerte está ligado al espacio del nacimiento de una nueva generación de ciudadanos, de hombres y mujeres. Al mismo tiempo, hay una necesidad de rememorar aquel momento donde aún existía un todo familiar. El proceso de escritura reconstruye ese tiempo anterior que es la construcción de un futuro en un espacio precario. ${ }^{8}$

Todo este proceso ya lo anunciaba Watanabe en Antígona, al hacernos ver que las consecuencias de la guerra estaban aún por venir. Efectivamente, Watanabe lo haría más explícito en el poema "En esa casa...", de su libro La piedra alada (2005):

Los ruidos de la muerte venían por el aire.

Nos respires, dijo alguien.

¿Fui yo el que habló? No lo sé, pero todos intuimos

que esa agonía

entraba en nosotros

como un oscuro veneno

que algún día tenemos que devolver. (2005: 48)

Efectivamente, el momento ha llegado.

\section{Referencias bibliográficas}

BERNALES, Enrique, y Carlos Villacorta (ed.) (2005). Los relojes se han roto. Antología de poesía peruana de los 90 . Guadalajara: Editorial Arlequín.

CHUECA, Luis Fernando (2001a): "Consagración de lo diverso: Una lectura de la poesía peruana de los noventa" en Lienzo 22, pp. 61-132.

CHUECA, Luis Fernando (2005b). Contemplación de los cuerpos. Lima: Estruendomudo.

CHUECA, Luis Fernando (2009c): “¿La hegemonía de lo conversacional? Notas para continuar una discusión” en Intermezzo Tropical 6-7, pp. 134-140.

COMISIÓN DE LA VERDAD Y RECONCILIACIÓN (2004). Hatun Willakuy: Versión abreviada del Informe Final de la Comisión de la Verdad y Reconciliación. Lima: Pontificia Universidad Católica del Perú.

CORNEJO POLAR, Antonio (1980). "La problematización del sujeto en la poesía conversacional”. En: Keith Mc Duffie y Rose Minc (editores) Homenaje a Alfredo A.

8 El poeta Jorge Frisancho también lo afirma de la siguiente manera: “y era nuestra casa el condenado / desierto del Perú" (48). Esa casa abierta es el espacio que se abre a todos los tiempos: al pasado, al presente, al futuro. 
Roggiano. En este aire de América. Pittsburgh: Instituto Internacional de Literatura Iberoamericana. pp. 201-207.

CRISÓLOGO, Roxana (2006). Ludy D. Lima: Flora Tristán.

DI LAURA, Giancarla. La influencia de César Vallejo en la poesía del Movimiento Kloaka, 19 de junio de 2016, 16.00h. http://letras.s5.com/cv140110.html.

FERNÁNDEZ, Manuel (2006). Octubre. Lima: Estruendomudo.

FRISANCHO, Jorge (2004). Desequilibrios. Lima: Pontificia Universidad Católica del Perú.

GUERRERO, Victoria (2005). Ya nadie incendia el mundo. Lima: Estruendomudo.

MORA, Tulio (1989). Cementerio general. Lima: Lluvia Editores.

ROMUALDO, Alejandro (2005). Ni pan, ni circo. Lima: Instituto Nacional de Cultura.

SILVA SANTISTEBAN, Rocío (2007). Las hijas del terror. Lima: COPE.

YRIGOYEN, José Carlos (2008). La hegemonía de lo conversacional. Lima: Lustra Editores.

WATANABE, José (2000a). Antígona. Lima: Yuyachkani / COMISEDH.

WATANABE, José (2005b) La piedra alada. Lima: Peisa.

ZARIQUIEY, Roberto (2005). Tratado de arqueología peruana. Lima: Pontificia Universidad Católica del Perú. 